\title{
Analytical Performance Prediction for Robust Constrained Model Predictive Control
}

\author{
Arthur Richards* and Jonathan P. How ${ }^{\dagger}$ \\ Massachusetts Institute of Technology, Cambridge, MA, 02139
}

\begin{abstract}
This paper presents a new analysis tool for predicting the closed-loop performance of a robust constrained Model Predictive Control (MPC) scheme. Currently, performance is typically evaluated by numerical simulation, leading to extensive computation when investigating the effect of controller parameters, such as horizon length, cost weightings, and constraint settings. The method in this paper avoids this computational burden, enabling a rapid study of the trades between the design parameters and performance. Previous work developed an MPC scheme employing constraint tightening to achieve robust feasibility and constraint satisfaction despite the action of an unknown but bounded disturbance. This paper shows that the expected performance of that scheme can be predicted using a combination of the gains of two linear systems, the optimal control for the unconstrained system, and a candidate policy used in performing the constraint tightening. The method also accounts for possible mismatch between the predicted level of disturbance and the actual level encountered. The predictions are compared with simulation results for several examples.
\end{abstract}

Keywords: Model Predictive Control, Robust Performance

\section{Introduction}

Model Predictive Control (MPC) $)^{5}$ is a promising technology for many aerospace applications. ${ }^{1,3,7,8}$ It uses the on-line solution of a numerical optimization problem and can readily accommodate hard constraints, such as relative position tolerances (or "error boxes") in spacecraft formation flight ${ }^{3}$ and collision avoidance in UAV guidance. ${ }^{1}$ Stability and robustness results for constrained MPC are well-addressed by existing work (see, for example, Ref. 6). However, robust performance of MPC is rarely considered analytically, but typically evaluated by numerical simulation instead. If various controller settings are to be investigated, this requires extensive computation. The contribution of this paper is a method of analytically predicting the performance of a system controlled by MPC, enabling rapid investigation of the effect of controller parameters without recourse to extensive simulation.

In Ref. 2, the authors describe a formulation for MPC offering robust feasibility, guaranteeing feasibility of each optimization and satisfaction of the constraints in the presence of an unknown but bounded disturbance. This method is an extension of an earlier work ${ }^{4}$ involving tightening constraints and retaining a "margin" in each plan for compensation against future disturbances. The margin is chosen such that a predetermined feedback policy can always be employed to counteract the disturbance, although in practice the on-line optimization usually finds a better solution than the application of that policy. While robust constraint satisfaction can imply bounds on the performance, e.g. the rate of fuel use of a spacecraft is clearly limited if its thrust at any time is limited, such bounds are very conservative. The new prediction method in this paper considers the expected performance.

Fig. 1 gives an overview of the performance prediction method. Fig. 1(a) shows the block diagram of the prediction method, in particular the roles of the actual and predicted disturbance levels. The predicted level is

*Dept. of Aeronautics and Astronautics, Student Member AIAA, arthurr@mit.edu

${ }^{\dagger}$ Associate Professor, Dept. of Aeronautics and Astronautics, Senior Member AIAA jhow@mit.edu 


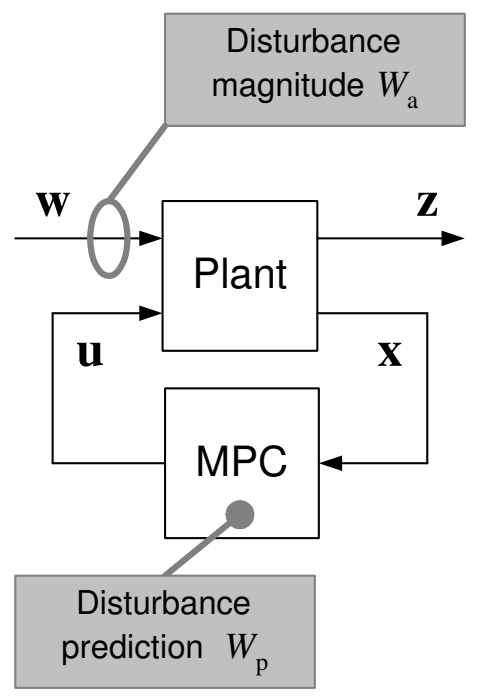

(a) Framework for Performance Prediction

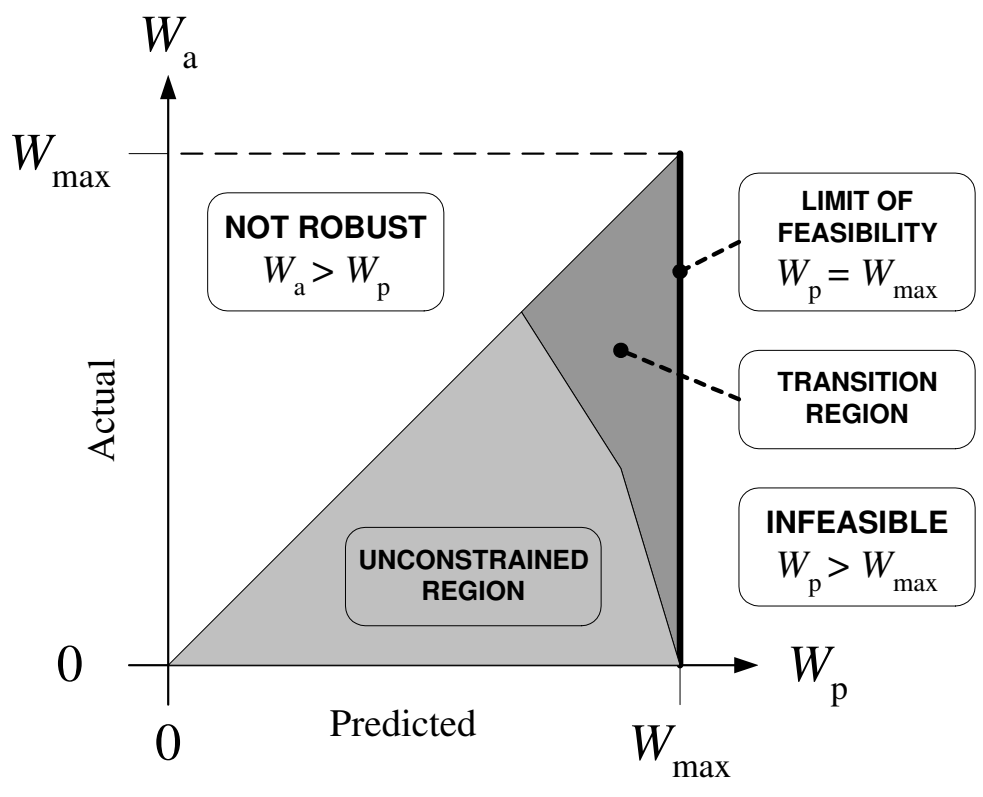

(b) Regions of Operation

Fig. 1: Overview of Performance Prediction. In Fig. 1(a), $\mathbf{z}$ is the performance output. In Fig. 1(b), $W_{\max }$ is the greatest level of predicted disturbance that allows a feasible optimization. The two shaded areas are those in which performance predictions can be made.

the designer's estimate of the actual disturbance level. It forms a disturbance model that is used to make the controller robust. The new performance analysis is based on two observations of the variation of performance over the space of actual and predicted disturbance levels, shown in Fig. 1(b). The first observation is that, if the actual disturbance is sufficiently low, corresponding to the lightly-shaded unconstrained region in Fig. 1(b), the controller behaves like the optimal finite-horizon regulator for the unconstrained system. This is not surprising in itself, but one of the contributions of this paper is a method of quantifying "sufficiently low" for this behavior. Observe that the upper limit of the unconstrained region varies with the predicted disturbance, as this determines the constraint tightening. The second observation is that, at the limit of feasibility, shown by the heavy line on the right of Fig. 1(b), the controller behaves like the predetermined candidate control used to determine the margin. Beyond the limit of feasibility, the constraint tightening means the problem is always infeasible, but right at the limit, it has only one solution available and its behavior is therefore predictable. Between the end of the unconstrained region and the limit of feasibility lies the transition region, shaded dark gray in Fig. 1(b). In this region, we approximate the performance by a smooth interpolation between the unconstrained behavior and the predetermined control policy. The key technical challenges of performing the prediction are determining, analytically, the limit of feasibility and the upper limit of the unconstrained region of operation.

The paper begins with a definition of the problem, in Section II. Section III reviews the robust MPC algorithm. The analytical prediction of performance is described in Section IV. Finally, Section V compares the performance predictions with numerical simulations for several examples.

\section{Problem Statement}

The aim is to control a linear system with discretized dynamics, a constrained output, performance output and disturbance input

$$
\begin{aligned}
\mathbf{x}(k+1) & =\mathbf{A x}(k)+\mathbf{B u}(k)+\mathbf{E w}(k) \\
\mathbf{y}(k) & =\mathbf{C x}(k)+\mathbf{D u}(k) \\
\mathbf{z}(k) & =\mathbf{F} \mathbf{x}(k)+\mathbf{G u}(k)
\end{aligned}
$$


where $\mathbf{x}(k) \in \Re^{N_{x}}$ is the state vector, $\mathbf{u}(k) \in \Re^{N_{u}}$ is the controlled input, $\mathbf{w}(k) \in \Re^{N_{w}}$ is the disturbance input, $\mathbf{y}(k) \in \Re^{N_{y}}$ is the constrained output and $\mathbf{z}(k) \in \Re^{N_{z}}$ is the performance output. The pair (A, B) is assumed to be controllable. The matrices $\mathbf{C}$ and $\mathbf{D}$ are chosen by the designer to form the outputs, which are constrained to remain within a bounded set

$$
\mathbf{y}(k) \in \mathcal{Y} \forall k
$$

This form of output constraints can capture both input and state constraints, or mixtures thereof, such as a limited control magnitude or an error requirement. The set $\mathcal{Y}$, also chosen by the designer, is a polytope defined by $N_{p}$ inequalities

$$
\mathcal{Y}=\left\{\mathbf{y} \mid \mathbf{p}_{n}^{T} \mathbf{y} \leq q_{n} \forall n \in 1 \ldots N_{p}\right\}
$$

The disturbance is unknown but bounded. It is to be assumed uncorrelated and uniformly distributed in a hypercube

$$
\mathbf{w}(k) \in \mathcal{B}_{\infty}\left(W_{a}\right) \forall k
$$

where

$$
\mathcal{B}_{\infty}(W)=\left\{\mathbf{w} \mid\|\mathbf{w}\|_{\infty} \leq W\right\}
$$

We do not assume that $W_{a}$ is known a priori: the disturbance model for control design, described in

Section III, uses a different value of the bound. Thus the prediction method considers the implication of inaccurate disturbance modeling.

\section{Robustly-Feasible MPC}

Define robust MPC optimization problem $\mathrm{P}\left(\mathbf{x}(k), W_{p}\right)$, starting from state $\mathbf{x}(k)$ with predicted disturbance bound $W_{p}$, as follows

$$
J^{*}\left(\mathbf{x}(k), W_{p}\right)=\min _{\mathbf{u}, \mathbf{x}, y} \sum_{j=0}^{N}\left(\mathbf{x}^{T}(k+j \mid k) \mathbf{Q} \mathbf{x}(k+j \mid k)+\mathbf{u}^{T}(k+j \mid k) \mathbf{R u}(k+j \mid k)\right)
$$

subject to

$$
\begin{aligned}
\forall j \in\{0 \ldots N\} & \\
\mathbf{x}(k+j+1 \mid k) & =\mathbf{A x}(k+j \mid k)+\mathbf{B u}(k+j \mid k) \\
\mathbf{y}(k+j \mid k) & =\mathbf{C x}(k+j \mid k)+\mathbf{D u}(k+j \mid k) \\
\mathbf{x}(k \mid k) & =\mathbf{x}(k) \\
\mathbf{x}(k+N+1 \mid k) & =\mathbf{0} \\
\mathbf{y}(k+j \mid k) & \in \mathcal{Y}\left(j ; W_{p}\right)
\end{aligned}
$$

where $N$ is the planning horizon. The choice of $N$ is the responsibility of the designer, but the analysis tool presented in this paper enables investigation of its effect on performance. $\mathbf{R}$ and $\mathbf{Q}$ are symmetric weighting matrices, with $\mathbf{Q}$ positive definite and $\mathbf{R}$ positive semi-definite. The set $\mathcal{Y}\left(j ; W_{p}\right)$ denotes the constraints on the $j^{\text {th }}$ predicted step, tightened to accommodate a predicted disturbance level $W_{p}$. Tightening is performed by the recursion

$$
\begin{aligned}
\mathcal{Y}\left(0 ; W_{p}\right) & =\mathcal{Y} \\
\mathcal{Y}\left(j+1 ; W_{p}\right) & =\mathcal{Y}\left(j ; W_{p}\right) \sim\left(\mathbf{C}+\mathbf{D} \mathbf{K}_{H}\right) \mathbf{L}_{H}(j) \mathbf{E} \mathcal{B}_{\infty}\left(W_{p}\right) \forall j \in\{0 \ldots N-1\}
\end{aligned}
$$

where the set $\mathcal{B}_{\infty}\left(W_{p}\right)$ is the predicted disturbance set. As discussed in Section II, the predicted disturbance limit $W_{p}$ is not assumed to be equal to the actual limit $W_{a}$. The operator $\sim$ denotes the Pontryagin difference $^{9}$ defined by

$$
\mathcal{A} \sim \mathcal{B}=\{\mathbf{a} \mid \mathbf{a}+\mathbf{b} \in \mathcal{A} \forall \mathbf{b} \in \mathcal{B}\}
$$

A Matlab routine is available ${ }^{10}$ for calculating the Pontryagin difference between two polytopes, and the result is also a polytope, with only the right-hand side of the inequalities changed, ${ }^{11}$ i.e. of the form

$$
\mathcal{Y}\left(j ; W_{p}\right)=\left\{\mathbf{y} \mid \mathbf{p}_{n}^{T} \mathbf{y} \leq q_{n}\left(j ; W_{p}\right) \forall n \in 1 \ldots N_{p}\right\}
$$


where the values of $q_{n}\left(j ; W_{p}\right)$ are determined by the Pontryagin difference algorithm. The controller $\mathbf{K}_{H}$ in (13) is chosen by the designer such that the static linear feedback control law $\mathbf{u}=\mathbf{K}_{H} \mathbf{x}$ makes the system nilpotent in at most $N$ steps. Also in (13), define $\mathbf{L}_{H}(j)$ as the state transition matrix for the closed-loop system under this control law

$$
\begin{aligned}
\mathbf{L}_{H}(0) & =\mathbf{I} \\
\mathbf{L}_{H}(j+1) & =\left(\mathbf{A}+\mathbf{B} \mathbf{K}_{H}\right) \mathbf{L}_{H}(j) \forall j \in\{0 \ldots N-1\}
\end{aligned}
$$

The problem defined above is employed in the following algorithm.

\section{Algorithm 1. (Robustly-Feasible MPC)}

1. Solve problem $\mathrm{P}\left(\mathbf{x}(k), W_{p}\right)$

2. Apply control $\mathbf{u}(k)=\mathbf{u}^{*}(k \mid k)$ from optimizing sequence

3. Go to 1

Theorem 1. (Robust Feasibility) If $\mathrm{P}\left(\mathbf{x}(0), W_{p}\right)$ has a feasible solution and $W_{p} \geq W_{a}$, then under the control of Algorithm 1 and the action of a disturbance obeying (4), all subsequent optimizations $\mathrm{P}\left(\mathbf{x}(k), W_{p}\right)$ are feasible.

Since the origin $\mathbf{0}$ is an invariant terminal constraint set and, for $W_{p} \geq W_{a}, \mathbf{w}(k) \in \mathcal{B}_{\infty}\left(W_{a}\right) \subseteq \mathcal{B}_{\infty}\left(W_{p}\right)$ then the proof of robust feasibility in Ref. 2 holds. For brevity, only an outline is given here. Assuming that a feasible solution is known for problem $\mathrm{P}\left(\mathbf{x}(k), W_{p}\right)$, then a solution can be constructed for problem $\mathrm{P}\left(\mathbf{x}(k+1), W_{p}\right)$ by shifting the previous solution by one step and adding a perturbation using controller $\mathbf{K}_{H}$ to accommodate the disturbance. Since this solution can be shown to be feasible for problem $\mathbf{P}\left(\mathbf{x}(k+1), W_{p}\right)$ for all disturbances obeying (4), it follows that the optimization itself is feasible. Thus feasibility at time $k$ implies feasibility at time $k+1$, and the theorem follows by recursion.

Remark 1. The formulation shown above is specialized to suit the performance analysis method developed in this paper. Robust feasibility can be proven under much more general conditions, ${ }^{2}$ with generalized cost function and terminal constraints, no requirement to find optimal solutions at each step, and without the assumption of any particular distribution of the disturbance within its bounding set.

\section{Analytical Performance Prediction}

The performance prediction is expressed in the following conjecture. The expected steady-state performance of a system (1a) under the control of Algorithm 1, expressed as the RMS value of the performance output (1c), for predicted and actual disturbances obeying

$$
0 \leq W_{a} \leq W_{p} \leq W_{\max }
$$

(the upper limit $W_{\max }$ is defined below) is approximated by

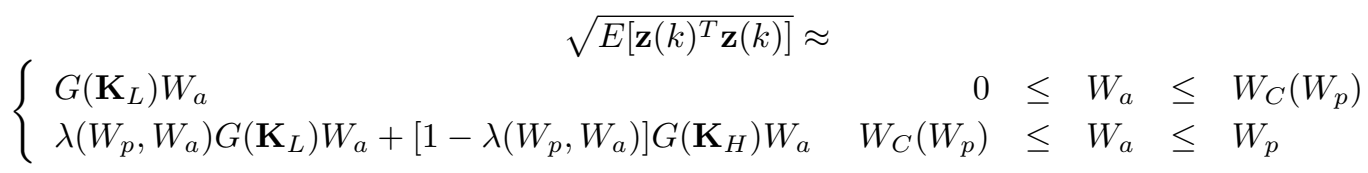

where

- $G(\mathbf{K})$ is the gain, from the infinity-norm of the disturbance to the two-norm of the output, of the system under a static, stabilizing, linear control $\mathbf{u}(k)=\mathbf{K} \mathbf{x}(k)$

- $\mathbf{K}_{L}$ is the finite-horizon LQR controller for the unconstrained system

- $W_{C}\left(W_{p}\right)$ is the level of actual disturbance at which the constraints, tightened for predicted disturbance $W_{p}$, begin to influence the performance. This defines the line separating the transition region and unconstrained region in Fig. 1(b). 
- $W_{\max }$ is the highest level of predicted disturbance $W_{p}$ that has a non-empty set of feasible initial states for the optimization $\mathrm{P}\left(\mathbf{x}(k), W_{p}\right)$

- $\lambda\left(W_{a}, W_{p}\right)$ is an interpolation function such that $\lambda\left(W_{C}\left(W_{p}\right), W_{p}\right)=0$ for any $W_{p}$ (i.e. on the dividing line between constrained and unconstrained regions in Fig. 1(b)) and $\lambda\left(W_{a}, W_{\max }\right)=1$ for any $W_{a} \neq 0$ (i.e. at the limit of feasibility). With this definition, the performance gain is $G\left(\mathbf{K}_{L}\right)$ at the edge of the unconstrained region and $G\left(\mathbf{K}_{H}\right)$ at the limit of feasibility.

The above is the mathematical expression of the observations expressed in Fig. 1(b). The following subsections describe how these quantities are calculated.

\section{A. Gain under Linear Control}

Under the assumptions concerning the disturbance made in Section II, the covariance matrix of the disturbance for unit input level $W_{a}=1$ is given by

$$
E\left[\mathbf{w}(k) \mathbf{w}^{T}(j)\right]=\left\{\begin{array}{rl}
\frac{1}{3} \mathbf{I} & k=j \\
\mathbf{0} & \text { otherwise }
\end{array}\right.
$$

Then, under the assumption that the state and input can be treated as normal random variables, the system gain is given by

$$
G(\mathbf{K})=\sqrt{\operatorname{tr}\left\{\mathbf{C}(\mathbf{K}) \mathbf{X}(\mathbf{K}) \mathbf{C}(\mathbf{K})^{T}\right\}}
$$

with $\mathbf{C}$ and $\mathbf{X}$ satisfying

$$
\begin{aligned}
& \mathbf{X}(\mathbf{K})=(\mathbf{A}+\mathbf{B K}) \mathbf{X}(\mathbf{K})(\mathbf{A}+\mathbf{B K})^{T}+\frac{1}{3} \mathbf{E E}^{T} \\
& \mathbf{C}(\mathbf{K})=\mathbf{F}+\mathbf{G K}
\end{aligned}
$$

where $\mathbf{X}(\mathbf{K})=E\left[\mathbf{x}(k) \mathbf{x}^{T}(k)\right]$ is the state covariance matrix under control $\mathbf{K}$ and can be found by solving the Lyapunov equation for its positive-definite solution. The validity of this analysis will be demonstrated by examples in Section V.

Remark 2. (Alternative Performance Metric) It may be desirable to express the performance in terms of a quadratic cost

$$
\sqrt{E\left[\mathbf{u}(k)^{T} \tilde{\mathbf{R}} \mathbf{u}(k)+\mathbf{x}(k)^{T} \tilde{\mathbf{Q}} \mathbf{x}(k)\right]}
$$

where $\tilde{\mathbf{Q}}$ and $\tilde{\mathbf{R}}$ are weighting matrices for the cost under investigation and need not match those used in the optimization cost (6). This can be achieved by setting

$$
\mathbf{F}=\left[\begin{array}{c}
\hat{\mathbf{Q}} \\
\mathbf{0}
\end{array}\right] \quad \mathbf{G}=\left[\begin{array}{c}
\mathbf{0} \\
\hat{\mathbf{R}}
\end{array}\right]
$$

using the Cholesky factors $\hat{\mathbf{Q}}^{T} \hat{\mathbf{Q}}=\tilde{\mathbf{Q}}$ and $\hat{\mathbf{R}}^{T} \hat{\mathbf{R}}=\tilde{\mathbf{R}}$.

\section{B. Unconstrained Control Solution}

The controller $\mathbf{K}_{L}$ is the unconstrained finite-horizon LQR solution, found by solving the following Ricatti equation

$$
\begin{aligned}
\mathbf{P}(N+1) & =\infty \mathbf{I} \\
\forall j \in\{0 \ldots(N+1)\} & \\
\mathbf{P}(j-1) & =\mathbf{Q}+\mathbf{A}^{T} \mathbf{P}(j) \mathbf{A}-\mathbf{A}^{T} \mathbf{P}(j) \mathbf{B}\left(\mathbf{R}+\mathbf{B}^{T} \mathbf{P}(j) \mathbf{B}\right)^{-1} \mathbf{B}^{T} \mathbf{P}(j) \mathbf{A} \\
\mathbf{K}(j-1) & =-\left(\mathbf{R}+\mathbf{B}^{T} \mathbf{P}(j) \mathbf{B}\right)^{-1} \mathbf{B}^{T} \mathbf{P}(j) \mathbf{A} \\
\mathbf{K}_{L} & =\mathbf{K}(0)
\end{aligned}
$$


It is also necessary to express the predicted outputs associated with the unconstrained solution as a function of the initial state $\mathbf{x}(k)$. Write

$$
\mathbf{y}(k+j \mid k)=\mathbf{H}(j) \mathbf{x}(k)
$$

where

$$
\mathbf{H}(j)=(\mathbf{C}+\mathbf{D K}(j)) \mathbf{L}(j)
$$

and the matrix $\mathbf{L}$ is the state transition matrix given by recursion

$$
\begin{aligned}
\mathbf{L}(0) & =\mathbf{I} \\
\mathbf{L}(j+1) & =(\mathbf{A}+\mathbf{B K}(j)) \mathbf{L}(j)
\end{aligned}
$$

These relations are used in Section D to find the limit of the unconstrained region.

\section{Limit of Feasibility}

The problem $\mathrm{P}\left(\mathbf{x}(k), W_{p}\right)$ becomes more constrained as the predicted disturbance $W_{p}$ is increased. The limit of feasibility $W_{\max }$ is defined as the greatest value of $W_{p}$ for which the set of feasible initial states for the problem $\mathrm{P}\left(\mathbf{x}(k), W_{p}\right)$ is non-empty. Since the terminal constraint is the origin, the problem will be infeasible if the origin is not contained in the tightest constraint set $\mathcal{Y}\left(N ; W_{p}\right)$ defined in (13). Therefore the limit of feasibility is found by solving the following optimization

$$
\begin{aligned}
W_{\max } & =\max W_{p} \\
\text { s.t. } \mathbf{0} & \in \mathcal{Y}\left(N ; W_{p}\right)
\end{aligned}
$$

By the definition of the Pontryagin difference (14), the condition

$$
\mathbf{0} \in \mathcal{A} \sim \mathcal{B}
$$

is equivalent to

$$
\mathbf{b} \in \mathcal{A} \forall \mathbf{b} \in \mathcal{B}
$$

Further, if $\mathcal{A}$ is convex and $\mathcal{B}$ is a mapping of a unit hypercube of dimension $N_{w}$ through a matrix $\mathbf{M}$, this can be expressed in terms of the vertices of $\mathcal{B}$

$$
\mathbf{M v}_{i} \in \mathcal{A} \forall i \in\left\{1 \ldots 2^{N_{w}}\right\}
$$

where $\mathbf{v}_{i}$ are the vertices of the unit hypercube, which can be evaluated straightforwardly. Extending this principle to the recursion for $\mathcal{Y}\left(N ; W_{p}\right)$ in (13) gives the following optimization to find $W_{\max }$

$$
\begin{aligned}
W_{\max }= & \max W_{p} \\
\text { s.t. } & W_{p} \sum_{j=0}^{(N-1)}\left(\mathbf{C}+\mathbf{D K}_{H}(j)\right) \mathbf{L}_{H}(j) \mathbf{E v}_{i_{j}} \in \mathcal{Y} \\
& \forall\left(i_{0}, i_{1} \ldots i_{(N-1)}\right) \in\left\{\left\{1 \ldots 2^{N_{w}}\right\} \times \ldots \times\left\{1 \ldots 2^{N_{w}}\right\}\right\}
\end{aligned}
$$

Observe that the constraint has to be evaluated for all combinations of vertices of the unit hypercube. Since $\mathcal{Y}$ is a polytope (3), this can be rewritten as

$$
W_{\max }=\min _{\left(n, i_{0}, i_{1} \ldots i_{(N-1)}\right) \in\left\{\left\{1 \ldots N_{p}\right\} \times\left\{1 \ldots 2^{N w}\right\} \times \ldots \times\left\{1 \ldots 2^{N_{w}}\right\}\right\}} \frac{q_{n}}{\mathbf{p}_{n}^{T} \sum_{j=0}^{(N-1)}\left(\mathbf{C}+\mathbf{D K}_{H}(j)\right) \mathbf{L}_{H}(j) \mathbf{E v}_{i_{j}}}
$$

which can be readily found, despite the large numbers of constraints to check. 


\section{Limit of Unconstrained Operation}

The quantity $W_{C}\left(W_{p}\right)$ is the greatest value of the actual disturbance $W_{a}$ for which the constraints, tightened for a predicted disturbance $W_{p}$, do not significantly influence the control performance. This corresponds to the division between the unconstrained region and the transition region, shown in Fig. 1(b). To calculate $W_{C}\left(W_{p}\right)$, we find the value of actual disturbance $W_{a}$ for which, if the closed-loop system behaves like the unconstrained optimal control $\mathbf{u}(k)=\mathbf{K}_{L} \mathbf{x}(k)$, then $95 \%$ of the solutions to the optimal unconstrained problem (26) satisfy the constraints. The choice of $95 \%$, as opposed to any other probability, is arbitrary and will be checked in simulations in Section V.

If the system behaves like the unconstrained optimal control, and assuming that the state can be treated as a Gaussian random variable, then with $95 \%$ probability, the state resides in an ellipsoid

$$
\frac{1}{W_{a}^{2}} \mathbf{x}^{T}(k) \mathbf{X}\left(\mathbf{K}_{L}\right)^{-1} \mathbf{x}(k) \leq 9
$$

where $\mathbf{X}\left(\mathbf{K}_{L}\right)$ is the state covariance matrix (20) under unconstrained control (25). This can be rewritten as a norm bound

$$
\|\mathbf{Y} \mathbf{x}(k)\|_{2} \leq 3 W_{a}
$$

where the matrix $\mathbf{Y}^{T} \mathbf{Y}=\mathbf{X}^{-1}\left(\mathbf{K}_{L}\right)$. Since $\mathbf{X}\left(\mathbf{K}_{L}\right)$ is taken to be the positive definite solution to the Lyapunov equation (20), the matrix $\mathbf{Y}$ exists and is invertible. Since the constraints (11) are of the form

$$
\mathbf{p}_{n}^{T} \mathbf{y}(k+j \mid k) \leq q_{n}\left(j ; W_{p}\right)
$$

we use (34) to derive a bound (for $95 \%$ probability) on the quantity $\mathbf{p}_{n}^{T} \mathbf{y}(k+j \mid k)$ as follows

$$
\begin{aligned}
\mathbf{p}_{n}^{T} \mathbf{y}(k+j \mid k) & =\mathbf{p}_{n}^{T} \mathbf{H}(j) \mathbf{x}(k) \\
& =\mathbf{p}_{n}^{T} \mathbf{H}(j) \mathbf{Y}^{-1} \mathbf{Y} \mathbf{x}(k) \\
& \leq 3 W_{a}\left\|\mathbf{Y}^{-T} \mathbf{H}(j)^{T} \mathbf{p}_{n}\right\|_{2}
\end{aligned}
$$

where $\mathbf{F}(j)$ are given by (26). Therefore, using the limits $q_{n}\left(j ; W_{p}\right)$ for the tightened constraints (15), the unconstrained solution is feasible, with $95 \%$ probability, if

$$
3 W_{a}\left\|\mathbf{Y}^{-T} \mathbf{H}(j)^{T} \mathbf{p}_{n}\right\| \leq q_{n}\left(j ; W_{p}\right) \forall n \in\left\{1 \ldots N_{P}\right\} \forall j \in\{0 \ldots N\}
$$

Then the value of $W_{C}\left(W_{p}\right)$ can be found by finding the greatest value of $W_{a}$ for which all conditions (35) hold, which can be done by simply checking each constraint and taking the minimum value

$$
W_{C}\left(W_{p}\right)=\min _{n \in\left\{1 \ldots N_{p}\right\} j \in\{0 \ldots N\}} \frac{q_{n}\left(j ; W_{p}\right)}{3\left\|\mathbf{Y}^{-T} \mathbf{H}(j)^{T} \mathbf{p}_{n}\right\|_{2}}
$$

\section{E. Interpolation Function}

The function $\lambda\left(W_{a}, W_{p}\right)$ is used in the transition region for interpolation between the unconstrained regime and the limit of feasibility

$$
\lambda=\left(\frac{W_{a}-W_{C}\left(W_{p}\right)}{W_{a}}\right)^{r}
$$

where $r>2$ gives a smooth transition away from the unconstrained performance. As $W_{p} \rightarrow W_{\max }$ then $W_{C}\left(W_{p}\right) \rightarrow 0$ hence also $\lambda \rightarrow 1$ for $W_{a} \neq 0$. Also, $\lambda \rightarrow 0$ as $W_{a} \rightarrow W_{C}\left(W_{p}\right)$.

Finally, the calculations described in the subsections above are combined in the following algorithm for performance prediction. 


\section{Algorithm 2. Performance Prediction}

Data: $\mathbf{A}, \mathbf{B}, \mathbf{C}, \mathbf{D}, \mathbf{E}, \mathbf{F}, \mathbf{G}, \mathcal{Y}, \mathbf{K}_{H}, N, \mathbf{Q}, \mathbf{R}, W_{a}, W_{p}$

1. If $W_{a}>W_{p}$, return 0 (not robust). Stop.

2. Calculate $W_{\max }$ using (31). If $W_{p}>W_{\max }$, return 0 (infeasible). Stop.

3. Calculate the unconstrained controller $\mathbf{K}_{L}$ using (25), gains $G\left(\mathbf{K}_{L}\right)$ using (19) and form the constraints $q_{n}\left(j ; W_{p}\right)(15)$ using $(12)$ and $(13)$

4. Calculate $W_{C}\left(W_{p}\right)$ using (36)

5. If $W_{a} \leq W_{C}\left(W_{p}\right)$, return $G\left(\mathbf{K}_{L}\right) W_{a}$ (unconstrained region). Stop.

6. Calculate gain $G\left(\mathbf{K}_{H}\right)$ and interpolation function $\lambda$ using (37) and return

$$
\lambda\left(W_{p}, W_{a}\right) G\left(\mathbf{K}_{L}\right) W_{a}+\left[1-\lambda\left(W_{p}, W_{a}\right)\right] G\left(\mathbf{K}_{H}\right) W_{a}
$$

\section{Examples}

This section demonstrates the performance prediction approximation (18) by comparing analytical predictions with simulation results for a variety of systems. The first two examples in this section investigate the variation of performance with actual and predicted disturbances, exploring the space shown in Fig. 1(b). The third example shows variation of performance with constraint limits, demonstrating the application of the analytical performance prediction to studying trades between design parameters.

Fig. 2 shows results for a neutrally-stable and unstable second-order system. The respective system $\mathbf{A}$ matrices are

$$
\mathbf{A}_{1}=\left[\begin{array}{ll}
1 & 1 \\
0 & 1
\end{array}\right] \quad \mathbf{A}_{2}=\left[\begin{array}{ll}
0.9 & 0.6 \\
0.2 & 0.5
\end{array}\right]
$$

with the following parameters common to both examples

$$
\mathbf{B}=\left[\begin{array}{l}
0.5 \\
1.0
\end{array}\right] \mathbf{C}=\left[\begin{array}{ll}
1 & 0 \\
0 & 1 \\
0 & 0
\end{array}\right] \mathbf{D}=\left[\begin{array}{c}
0 \\
0 \\
0.5
\end{array}\right] \mathbf{E}=\left[\begin{array}{cc}
0.5 & 0 \\
0 & 1
\end{array}\right]
$$

The constraints are a unit bound on the infinity norm i.e. a unit magnitude bound on each element

$$
\|\mathbf{y}(k)\|_{\infty} \leq 1
$$

The optimization costs and performance metric, using the form in Remark 2, are identical, penalizing the control energy

$$
\tilde{\mathbf{Q}}=\mathbf{Q}=\mathbf{I}_{2} \quad \tilde{\mathbf{R}}=\mathbf{R}=100
$$

The problem was solved with a horizon of $N=6$ steps. The expected disturbance $W_{p}$ and actual disturbance $W_{a}$ were varied between 0 and the appropriate $W_{\max }$, precalculated for each system. In the prediction results (Figs. 2(a) and 2(c)), the unconstrained region is white and the transition region shaded gray. In both cases, the predictions closely match the results of the simulations.

Fig. 3 shows an example in which the constraint levels are varied. The example uses the neutrally-stable system matrix $\mathbf{A}_{1}$. The predicted disturbance $W_{p}$ is fixed at $80 \%$ of the maximum $W_{\max }$ and the actual disturbance $W_{a}$ at $80 \%$ of $W_{p}$. The constraints are

$$
\left|y_{1}(k)\right| \leq Y_{1} \quad\left|y_{2}(k)\right| \leq Y_{2} \quad\left|y_{3}(k)\right| \leq 1
$$

where $Y_{1}$ and $Y_{2}$ are variable limits on the position and velocity, respectively, whose effect is to be investigated. In the transition region, shaded gray in Fig. 3(a), the exact level of performance is underestimated by the prediction method. However, the predictions still capture important trends in the performance. The limiting values of $Y_{1}$ and $Y_{2}$ at which the constraints become significant are accurately predicted, as are the limits of feasibility. Also, the prediction correctly identifies regions in which the performance depends on $Y_{1}$ but not $Y_{2}$ and vice versa, as well as the line of transition between. Hence it can be seen that the prediction method allows the designer to assess the impact of design decisions such as constraint settings. 


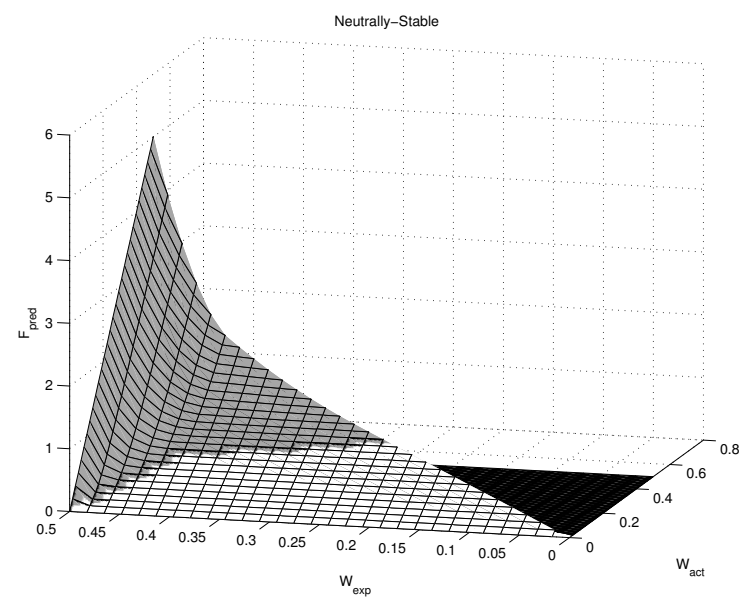

(a) Predicted, Neutrally-Stable $\mathbf{A}_{1}$

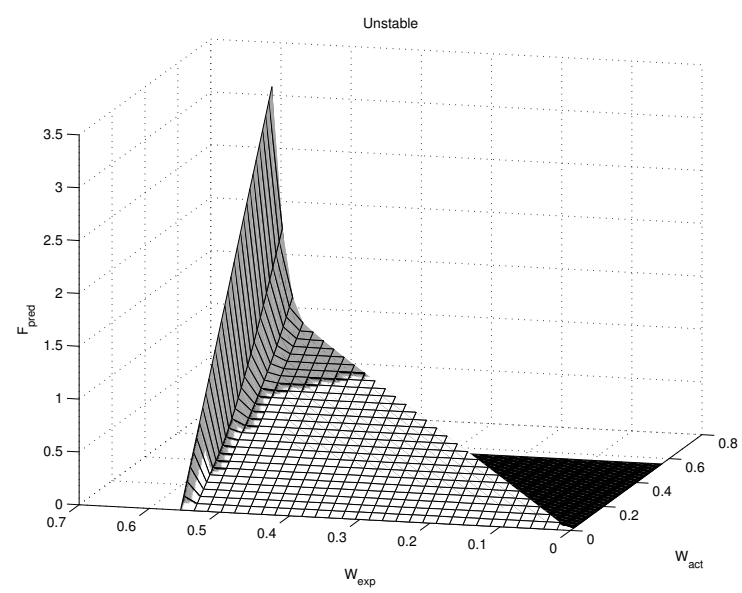

(c) Predicted, Unstable $\mathbf{A}_{2}$

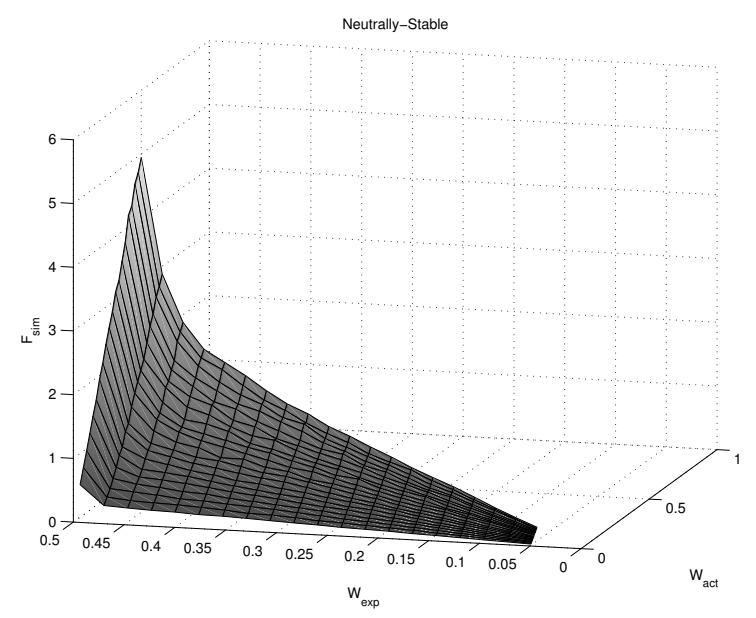

(b) Simulated, Neutrally-Stable $\mathbf{A}_{1}$

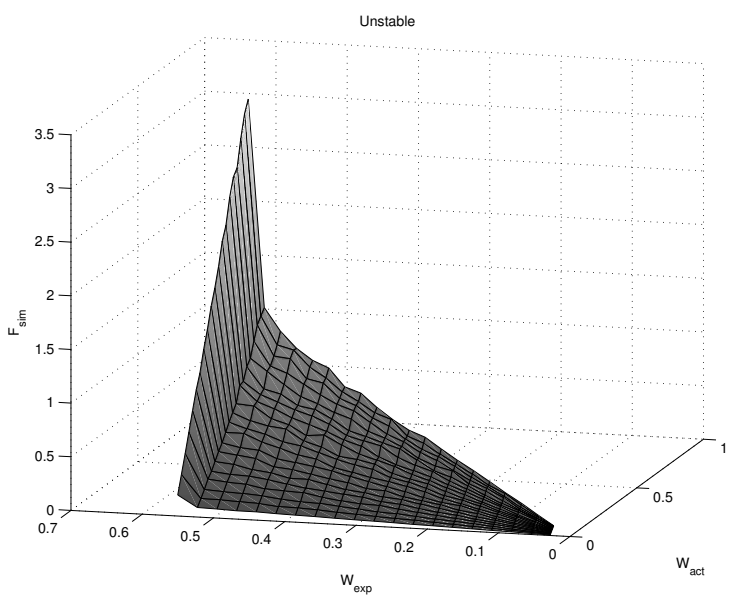

(d) Simulated, Unstable $\mathbf{A}_{2}$

Fig. 2: Comparison of Predicted Performance and Simulation Results for Two Systems with Varying Expected and Actual Disturbances. In Figs. 2(a) and 2(c), the unconstrained region is in white and the transition region in gray.

\section{Conclusion}

This paper has presented a method of analytically predicting the expected closed-loop performance of a system using a form of robust Model Predictive Control. This enables trade studies on the effect of controller parameters to be performed without extensive numerical simulation. The prediction method has been demonstrated for several example systems and compared to simulation results. It has been shown to correctly identify trends in performance as a function of disturbance levels and constraint settings.

\section{Acknowledgments}

This work was funded under Cooperative Agreement NCC5-729 through the NASA GSFC Formation Flying NASA Research Announcement. Any opinions, findings, and conclusions or recommendations expressed in this material are those of the author(s) and do not necessarily reflect the views of the National Aeronautics and Space Administration. 


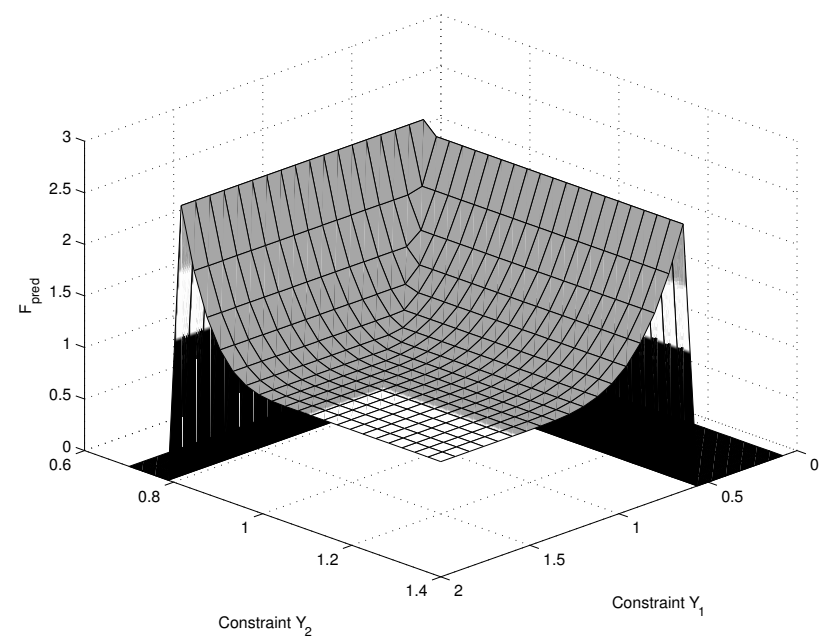

(a) Predicted

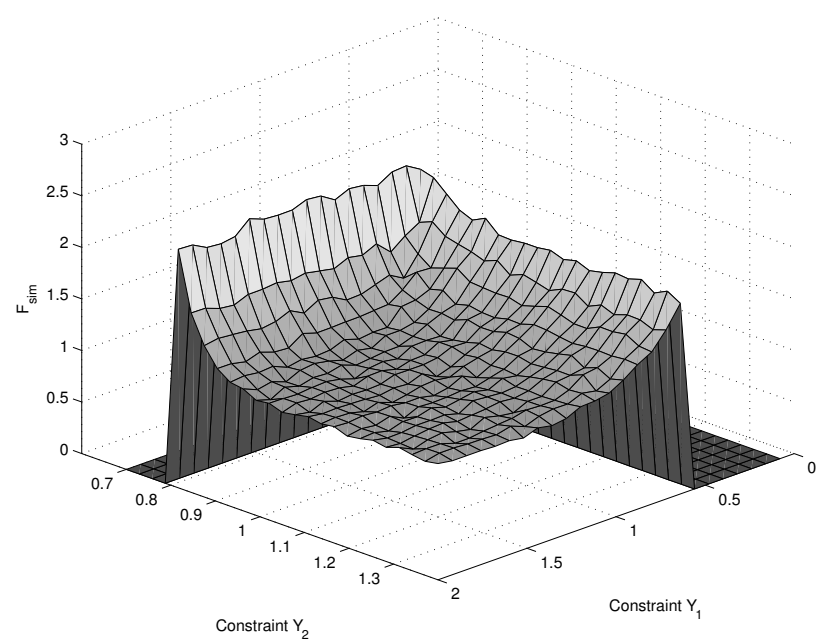

(b) Simulated

Fig. 3: Comparison of Predicted Performance and Simulation Results for a System with Varying Constraints

\section{References}

${ }^{1}$ A. G. Richards and J. P. How, "Model Predictive Control of Vehicles Maneuvers with Guaranteed Completion Time and Robust Feasibility," American Control Conference, Denver CO, AACC, 2003.

${ }^{2}$ A. G. Richards and J. P. How, "A Computationally-Efficient Technique for Robust Model Predictive Control," submitted to IEEE Transactions on Automatic Control, May 2004. Draft available from authors by request.

${ }^{3}$ G. Inalhan, J. P. How and M. Tillerson, "Co-ordination and control of distributed spacecraft systems using convex optimization techniques," International Journal of Robust and Nonlinear Control, Vol.12, , John Wiley \& Sons, 2002, p.207242.

${ }^{4}$ J. R. Gossner, B. Kouvaritakis and J. A. Rossiter, "Stable Generalized Predictive Control with Constraints and Bounded Disturbances," Automatica, Vol 33 No 4, Pergamon Press, UK, 1997, p.551.

${ }^{5}$ J. M. Maciejowski, Predictive Control with Constraints, Prentice Hall, England, 2002.

${ }^{6}$ D. Q. Mayne, J. B. Rawlings, C. V. Rao, P. O. M. Scokaert, "Constrained Model Predictive Control: Stability and Optimality," Automatica, 36(2000), Pergamon Press, UK, pp. 789-814.

${ }^{7}$ V. Manikonda, P. O. Arambel, M. Gopinathan, R. K. Mehra and F. Y. Hadaegh, "A Model Predictive Control-based Approach for Spacecraft Formation Keeping and Attitude Control," ACC, San Diego CA, June 1999.

${ }^{8}$ W. Dunbar and R. Murray, "Model predictive control of coordinated multi-vehicle formations," Conference on Decision and Control, IEEE, 2002.

${ }^{9}$ I. Kolmanovsky and E. G. Gilbert, "Maximal Output Admissible Sets for Discrete-Time Systems with Disturbance Inputs," American Control Conference, Seattle WA, , AACC, 1995, p.1995.

${ }^{10}$ E. Kerrigan, Invariant Set Toolbox for Matlab, available at http://www-control.eng.cam.ac.uk/eck21, July 2003.

${ }^{11}$ E. C. Kerrigan, "Robust Constraint Satisfaction: Invariant Sets and Predictive Control," PhD Thesis, Cambridge University, November 2000. 